\title{
The Quebec Parkinson Network: A Researcher-Patient Matching Platform and Multimodal Biorepository
}

Ziv Gan-Or ${ }^{\mathrm{a}, \mathrm{b}, \mathrm{c}, *}$, Trisha Rao ${ }^{\mathrm{d}}$, Etienne Leveille ${ }^{\mathrm{b}, \mathrm{e}}$, Clotilde Degroot $^{\mathrm{a}, \mathrm{b}}$, Sylvain Chouinard $^{\mathrm{f}}$, Francesca Cicchetti $^{\mathrm{g}, \mathrm{h}}$, Alain Dagher ${ }^{\mathrm{b}}$, Samir Das ${ }^{\mathrm{i}}$, Alex Desautels ${ }^{\mathrm{j}, \mathrm{k}}$, Janelle Drouin-Ouellet ${ }^{\mathrm{l}}$, Thomas Durcan $^{\mathrm{a}, \mathrm{b}}$, Jean-François Gagnon ${ }^{\mathrm{j}, \mathrm{m}}$, Angela Genge ${ }^{\mathrm{a}, \mathrm{b}, \mathrm{d}}$, Jason Karamchandani ${ }^{\mathrm{n}}$, Anne-Louise Lafontaine ${ }^{\mathrm{a}, \mathrm{b}, \mathrm{o}}$, Sonia Lai Wing Sun ${ }^{\mathrm{b}}$, Mélanie Langlois ${ }^{\mathrm{p}, \mathrm{q}}$, Martin Levesque ${ }^{\mathrm{h}, \mathrm{r}}$, Calvin Melmed $^{\mathrm{a}, \mathrm{s}}$, Michel Panisset ${ }^{\mathrm{f}}$, Martin Parent ${ }^{\mathrm{h}, \mathrm{r}}$, Jean-Baptiste Poline ${ }^{\mathrm{b}}$, Ronald B. Postuma ${ }^{\mathrm{a}, \mathrm{b}}$, Emmanuelle Pourcher ${ }^{\mathrm{p}, \mathrm{q}}$, Guy A. Rouleau ${ }^{\mathrm{a}, \mathrm{b}, \mathrm{c}}$, Madeleine Sharp ${ }^{\mathrm{a}, \mathrm{b}}$, Oury Monchi ${ }^{\mathrm{a}, \mathrm{t}, \mathrm{u}}$, Nicolas Duprée,q and Edward A. Fon ${ }^{\mathrm{a}, \mathrm{b}, *}$

${ }^{a}$ Department of Neurology and Neurosurgery, McGill University, Montréal, QC, Canada

${ }^{\mathrm{b}}$ Montreal Neurological Institute, McGill University, Montréal, QC, Canada

${ }^{\mathrm{c}}$ Department of Human Genetics, McGill University, Montréal, QC, Canada

${ }^{\mathrm{d}}$ Clinical Research Unit, Montreal Neurological Institute, McGill University, Montréal, QC, Canada

${ }^{\mathrm{e}}$ Faculty of Medicine, McGill University, Montréal, QC, Canada

${ }^{\mathrm{f}}$ Unité des trouves du mouvement André Barbeau, Centre hospitalier de l'Université de Montréal, Montreal, $Q C$, Canada

${ }^{\mathrm{g}}$ Centre de Recherche du CHU de Québec, Axe Neurosciences, Québec, QC, Canada

${ }^{\mathrm{h}}$ Département de Psychiatrie \& Neurosciences, Université Laval, Québec, QC, Canada

${ }^{\mathrm{i}}$ McGill Centre for Integrative Neuroscience, Montreal Neurological Institute, Montreal, QC, Canada

${ }^{\mathrm{j} C e n t r e ~ d ' E ́ t u d e s ~ A v a n c e ́ e s ~ e n ~ M e ́ d e c i n e ~ d u ~ S o m m e i l ~ a n d ~ N e u r o l o g y ~ S e r v i c e, ~ H o ̂ p i t a l ~ d u ~ S a c r e ́-C \emptyset e u r ~ d e ~}$ Montréal, Montréal, QC, Canada

${ }^{\mathrm{k}}$ Department of Neurosciences, Université de Montréal, Montréal, QC, Canada

${ }^{1}$ Faculty of Pharmacy, Université de Montréal, Montreal, Quebec, QC, Canada

${ }^{\mathrm{m}}$ Department of Psychology, Université du Québec à Montréal, Montreal, QC, Canada

${ }^{\mathrm{n}}$ Department of Pathology, Montreal Neurological Institute, McGill University, Montréal, QC, Canada

${ }^{\circ}$ Department of Neurology, McGill University Medical Centre, Montréal, QC, Canada

${ }^{\mathrm{p}}$ Division of Neurosciences, CHU de Québec, Université Laval, Québec City, QC, Canada

${ }^{\mathrm{q}}$ Department of Medicine, Faculty of Medicine, Université Laval, Québec City, QC, Canada

${ }^{\mathrm{r}}$ CERVO Brain Research Centre, Québec City, QC, Canada

${ }^{\mathrm{s}}$ Jewish General Hospital, McGill University, Montréal, QC, Canada

${ }^{\mathrm{t}}$ Departments of Clinical Neurosciences and Radiology, University of Calgary, AB, Canada

${ }^{\mathrm{u}}$ Hotchkiss Brain Institute, Cumming School of Medicine, University of Calgary, AB, Canada

Accepted 27 October 2019

\footnotetext{
${ }^{*}$ Correspondence to: Ziv Gan-Or, Montreal Neurological Institute, McGill University, 1033 Pine Avenue, West, Ludmer Pavilion, room 312, Montreal, QC, H3A 1A1, Canada. Tel.: +1 514 398 5845; E-mail: ziv.gan-or@mcgill.ca. and Edward A. Fon,
}

Montreal Neurological Institute, McGill University, 3801 Rue Université, Montréal, QC H3A 2B4, Canada. Tel.: +1 514398 8398; E-mail: ted.fon@mcgill.ca. 


\begin{abstract}
.
Background: Genetic, biologic and clinical data suggest that Parkinson's disease (PD) is an umbrella for multiple disorders with clinical and pathological overlap, yet with different underlying mechanisms. To better understand these and to move towards neuroprotective treatment, we have established the Quebec Parkinson Network (QPN), an open-access patient registry, and data and bio-samples repository.

Objective: To present the QPN and to perform preliminary analysis of the QPN data.

Methods: A total of 1,070 consecutively recruited PD patients were included in the analysis. Demographic and clinical data were analyzed, including comparisons between males and females, PD patients with and without RBD, and stratified analyses comparing early and late-onset PD and different age groups.

Results: QPN patients exhibit a male:female ratio of 1.8:1, an average age-at-onset of 58.6 years, an age-at-diagnosis of 60.4 years, and average disease duration of 8.9 years. REM-sleep behavior disorder (RBD) was more common among men, and RBD was associated with other motor and non-motor symptoms including dyskinesia, fluctuations, postural hypotension and hallucinations. Older patients had significantly higher rates of constipation and cognitive impairment, and longer disease duration was associated with higher rates of dyskinesia, fluctuations, freezing of gait, falls, hallucinations and cognitive impairment. Since QPN's creation, over 60 studies and 30 publications have included patients and data from the QPN.

Conclusions: The QPN cohort displays typical PD demographics and clinical features. These data are open-access upon application (http://rpq-qpn.ca/en/), and will soon include genetic, imaging and bio-samples. We encourage clinicians and researchers to perform studies using these resources.
\end{abstract}

Keywords: Parkinson disease, Quebec Parkinson Network, registry, biobank

\section{INTRODUCTION}

Parkinson's disease (PD) is a common neurodegenerative, age-related movement disorder with a prevalence of $1-2 \%$ in individuals older than 60 years of age [1]. As the world's population ages, the number of PD patients and its burden are projected to dramatically increase in the next decades [2]. The etiology of PD is complex, and includes environmental, genetic and aging-related factors [3]. Furthermore, various genetic, biologic and clinical data suggest that PD is in fact not a single disease, but an umbrella for multiple disorders with clinical and pathological overlap, yet likely with different underlying biological mechanisms. For example, PD patients with $G B A$ mutations (GBA-PD) and PD patients with $L R R K 2$ mutations (LRRK2-PD) may represent distinct subtypes of PD, in which the typical clinical course and the underlying mechanisms may be different [4-7]. Accordingly, future treatment will likely be directed towards the specific mutations and mechanisms in each of these forms of PD [8]. Similarly, clustering based on clinical variables have demonstrated that clinical subtypes can be distinguished based on baseline symptoms that predict the clinical outcome of PD $[9,10]$. A better understanding of this disease and its underlying mechanisms are critical at this point if we wish to identify efficient neuroprotective or neurorestorative treatments.

Recent initiatives, such as the Parkinson's Progression Marker Initiative (PPMI) and other similar cohorts have emerged [11], to better define and understand PD and its subtypes, identify reliable biomarkers and consequently accelerate therapeutic development. Herein, we present a preliminary analysis of the Quebec Parkinson Network (QPN) patient registry; a longitudinal cohort comprised of individuals with PD recruited in the province of Quebec, Canada. The registry is continuously updated with longitudinal follow-up information and with newly recruited patients. Available information includes demographic data such as age, sex, mother tongue, level of education, smoking status, coffee consumption, as well as clinical data such as presenting and current motor symptoms, non-motor symptoms, Hoehn and Yahr stage, medications. Genetics, imaging and additional clinical measures are currently being collected and will be added soon. This is an open-access cohort, which allows clinicians and researchers, upon application, to access patient data, imaging and samples, in accordance with the open access policy of the Montreal Neurological Institute (MNI) Tanenbaum Open Science Institute (TOSI) [12]. Herein, we present preliminary data analysis from the QPN, and introduce the upcoming Canadian Open Parkinson's Network (C-OPN), a patient registry inspired by the QPN model that will recruit patients across all Canadian provinces.

\section{METHODS}

\section{Population and data collection}

At the time of collecting the data for this paper (January 2019), a total of 1,070 PD patients were enrolled 
Table 1

Demographic and clinical variables collected in the Quebec Parkinson Network

\begin{tabular}{ll}
\hline Demographic data & Clinical data \\
\hline - Sex & - Date of symptom onset \\
- Date of birth & - Date of diagnosis \\
- Laterality & - Confirmation of diagnosis \\
- First language, spoken language, number of languages & - Atypical Parkinsonian syndromes (MSA, PSP, CBD, DLB, \\
spoken & FTD, ET) \\
- Residence (house, institution, other) & - Modified Hoehn and Yahr stage \\
- Situation (single, in relationship, other caregiver) & - Asymmetry of symptoms \\
- Education & - First and dominant symptoms (tremor, rigidity, bradykinesia, postural \\
- Smoking history & instability) \\
- Alcohol and coffee consumption & - Presence of dyskinesia, motor fluctuations, motor block \\
- Occupation and home location (urban, rural) & - History of fall \\
- Environmental exposure to neurotoxins & - Anti-PD treatment history \\
- Frequency of head trauma & - RBD and sleep disorder \\
- MRI compatibility & - Comorbidities (hypertension, hypotension, diabetes, constipation) \\
- Family history of PD and PD-related disease & - Presence and treatment of psychiatric disorders (anxiety, depression, \\
& - bipolar disorder, hallucinations, impulsive/compulsive behaviour) \\
& - Presence of dementia \\
\hline
\end{tabular}

CBD, corticobasal degeneration; DLB, dementia with Lewy bodies; ET, essential tremor; FTD, frontotemporal dementia; MSA, multiple system atrophy; PD, Parkinson's disease; PSP, progressive supranuclear palsy; RBD, REM (rapid eye movement) sleep behaviour disorder. ${ }^{\text {a }}$ Stages are described in Supplementary Table 1.

into the QPN and analysis is presented on this cohort of 1,070 patients. However, enrolment is ongoing, with a total of $>1,400$ patients recruited by August 2019 at the time of completing the first draft of this manuscript. All patients were diagnosed by a movement disorder specialist in the province of Quebec according to the MDS criteria or previously published criteria for patients who were recruited before the publication of the MDS criteria [13]. We included here all the patients, i.e., clinically established and clinically probable based on the MDS criteria, and PD patients diagnosed using previous criteria such as the UK Brain Bank criteria. The majority of patients (76.9\%) were recruited at the MNI, the Centre Hospitalier de l'Université de Montréal (CHUM), and the Centre Hospitalier Universitaire de Québec (CHUQ). Data were collected by using the QPN Questionnaire (Supplementary Material), which is completed for each participant with the help of a neurologist or a trained research assistant. This questionnaire collects data on demographics, diagnosis, motor symptoms, treatment and medications, lifestyle and environmental risk factors, magnetic resonance imaging (MRI) compatibility, sleeping habits, family history of PD or PD-related disorders, non-motor symptoms and comorbidities, as well as additional tests such as cognitive and psychiatric evaluations (Table 1). More details on the type of tests used can be found in the Supplementary Material. The QPN categorizes the collected data according to criteria commonly used for PD clinical trials. Longitudinal clinical data are being collected from consenting patients every 18 months. Since most of the patients were recruited in the last 18 months, longitudinal data are currently available for a small portion of the patients. While not analysed in the current study, these data will be available for future studies.

Patients recruited through the QPN Participant Registry are automatically given the option to be included in the Neuro Open Science Clinical Biologic Imaging and Genetic Repository (NeurO C-BIGR), a multi-modal bio-repository of the MNI (https://www.mcgill.ca/c-bigneuro/). The workflow of this process is depicted in Fig. 1. The objective of this ongoing project is to collect blood from all participants in the registry for genotyping/sequencing and for the generation of patient-specific induced pluripotent stem cell (iPSC) lines. In addition, during the first phase of the QPN, 200 of these participants will also undergo magnetoencephalography (MEG), MRI, a neuropsychological evaluation, a motor evaluation, and provide a recorded speech sample.

All patients recruited through the QPN have signed an informed consent form at enrollment, and the study protocol was approved by the institutional research ethics board.

\section{Statistical analysis}

Data are presented as percentages for categorical variables or as averages \pm standard deviation $(\mathrm{SD})$ for 


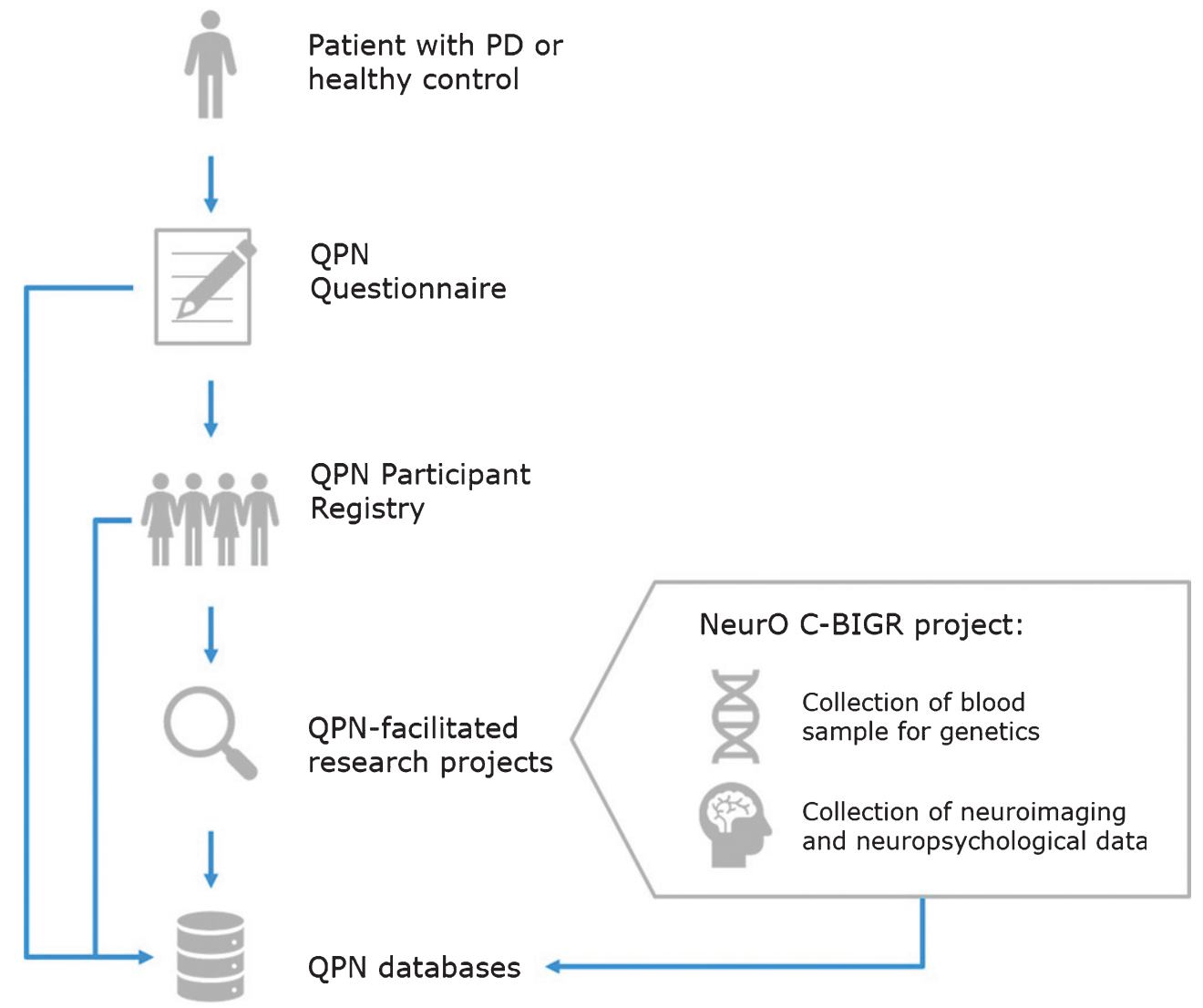

Fig. 1. Quebec Parkinson Network (QPN) workflow for collection of patient data. Patients with PD or healthy controls who are recruited to the QPN Participant Registry must complete the QPN Questionnaire. Active members of the QPN Participant Registry may be selected for participation in QPN-facilitated research projects, such as the NeurO C-BIGR project which involves collection of blood samples for genetics and iPSCs derivation, as well as neuroimaging and neuropsychological data. Patient data collected through the QPN Questionnaire, medical records of patients in the QPN Participant Registry, and obtained via QPN-facilitated research projects are entered into the QPN databases.

continuous variables. To compare frequencies of categorical variables, either $\chi^{2}$ test or Fisher Exact test were used. If adjustment for covariates was required, binary logistic regression models were used. To compare continuous variables, T-test or analysis of variance (ANOVA) were used, and when adjustment for covariates was required, we used linear regression models.

Sub-group and stratified analyses were performed to examine different correlations between sub-groups and PD demographic and clinical characteristics. These included sex-stratified analysis (including 691 males and 379 females), PD patients with and without probable rapid eye movement (REM) sleep behavior disorder (RBD), determined by the validated RBD1Q [14] (PD+RBD, $n=381$, PD-RBD, $n=570$, respectively), stratification by age groups $(0-50$ years, $n=45,51-60$ years, $n=171,61-70$ years, $n=371$,
$71-80$ years, $n=362$, and $>80$ years, $n=107)$, disease duration (0-5 years, $n=194), 6-10$ years, $n=163$, and $>10$ years, $n=159)$ and early-onset PD (EOPD, defined as age at onset [AAO] under 50 years, $n=108$ ) vs late-onset PD (LOPD, 50 years or above, $n=410$ ). Bonferroni correction for multiple comparisons was performed as required. All statistical analyses were performed using SPSS 23.0 (IBM Inc.).

\section{RESULTS}

\section{Demographic and clinical characteristics of patients in the Quebec Parkinson Network}

Figures 2 and 3 depict the main demographic and clinical characteristics of the first 1,070 PD patients from the QPN Participant Registry. Supplementary Tables 2 and 3 include more detailed data on the main 

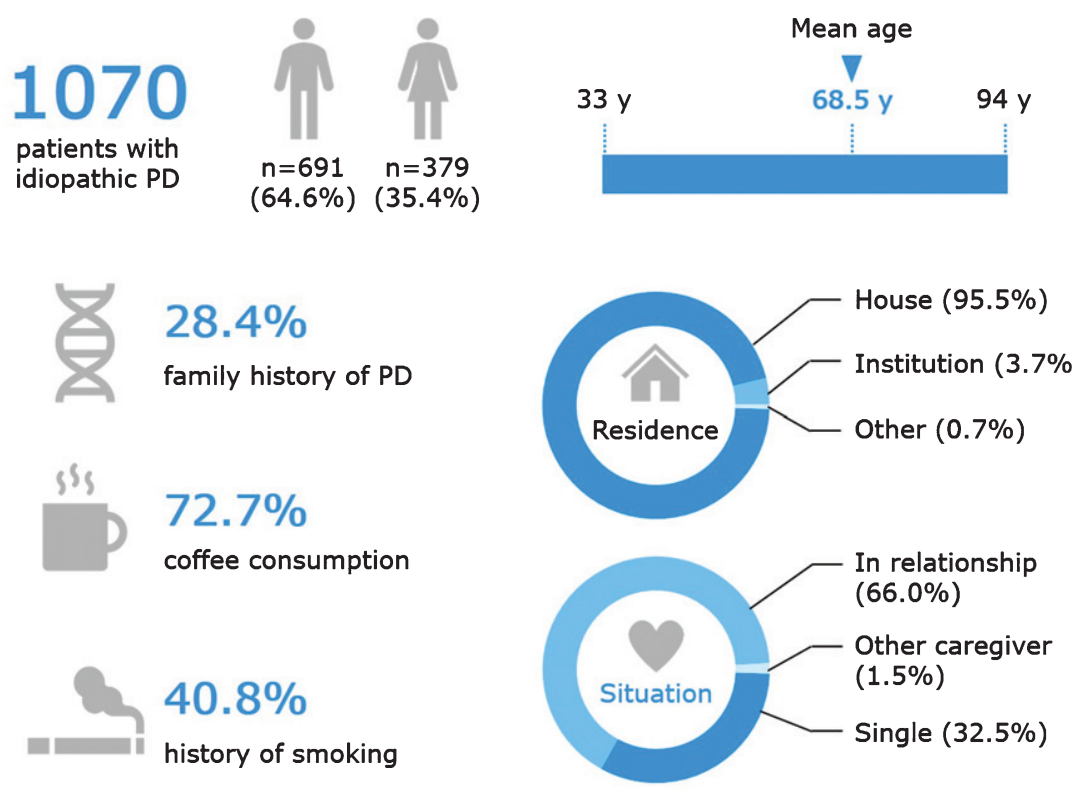

Fig. 2. Key demographic characteristics of patients in the Quebec Parkinson Network cohort. Data are presented for the 1070 patients with PD, except the following for which data were not available for all patients: age $(n=1056)$ and coffee consumption $(n=985)$. Family history of PD refers to having a mother, father, sibling, child, or other relation (uncle, aunt, cousin, niece, or nephew) with PD or PD-related disorder. Coffee consumption refers to current coffee drinkers and does not include former coffee drinkers. A history of smoking refers to current and former smokers.

demographic and clinical variables. This cohort is typical of other PD tertiary and academic centers' patient cohorts, with a male:female ratio of about $1.8: 1$, average AAO of $58.6 \pm 11.3$ years (available data for $n=518$ ), age at diagnosis of $60.4 \pm 11.0$ years (available data for $n=921$ ) and average current age of $68.5 \pm 9.8$ years (available data for $n=1056$ ). The average disease duration is $8.9 \pm 6.8$ years (available data for $n=516$ ), with an average Hoehn \& Yahr score of $2.35 \pm 0.88$ (available data for $n=637$ ). Of the 518 patients for whom data on AAO was available, 108 (20.9\%) had EOPD. Supplementary Table 3 details other motor and non-motor clinical characteristics of the QPN cohort, such as probable RBD (reported in $40.1 \%$ of patients with available data, $n=951$ ), self-reported dyskinesia (47.2\%, available data for $n=835)$ and freezing of gait $(16.2 \%$, available data for $n=1070$ ). Non-motor symptoms are particularly prevalent, with self-reported anxiety being the most common $(56.9 \%$, available data for $n=865)$.

\section{Sex differences in the clinical presentation of $P D$}

To examine whether specific clinical characteristics were associated with sex, we performed a sex-stratified analysis comparing male $(n=691)$ and female $(n=379)$ patients with PD (Table 2). There were no statistically significant differences in age, AAO, age at diagnosis, time to diagnosis, and Hoehn \& Yahr stage between male and female patients. Female patients had a longer disease duration than males, with nominal significance $(9.71 \pm 7.58$ years vs $8.43 \pm 6.23$ years, respectively, $p=0.047)$. The difference was not statistically significant after correction for multiple comparisons or after adjustment for age of onset, which was younger in females, although not statistically significant $(57.45 \pm 12.03$ years vs $59.24 \pm 10.73$ years, respectively, $p=0.08$ ).

When compared to male patients, tremor, postural hypotension, anxiety, dyskinesia and asymmetry of symptoms were more prevalent in females (Table 2). None of these associations were statistically significant after correction for multiple comparisons (Bonferroni-corrected $p$ value of 0.0025 ) or after adjustment for disease duration, except for dyskinesia which remained statistically significant after age and/or disease duration adjustment, but not after Bonferroni correction. The only association that remained statistically significant after both Bonferroni correction and adjustment for age and/or disease duration was probable RBD, which was more common in males $(44.2 \%$ vs $32.7 \%, p<0.001)$. There were no 

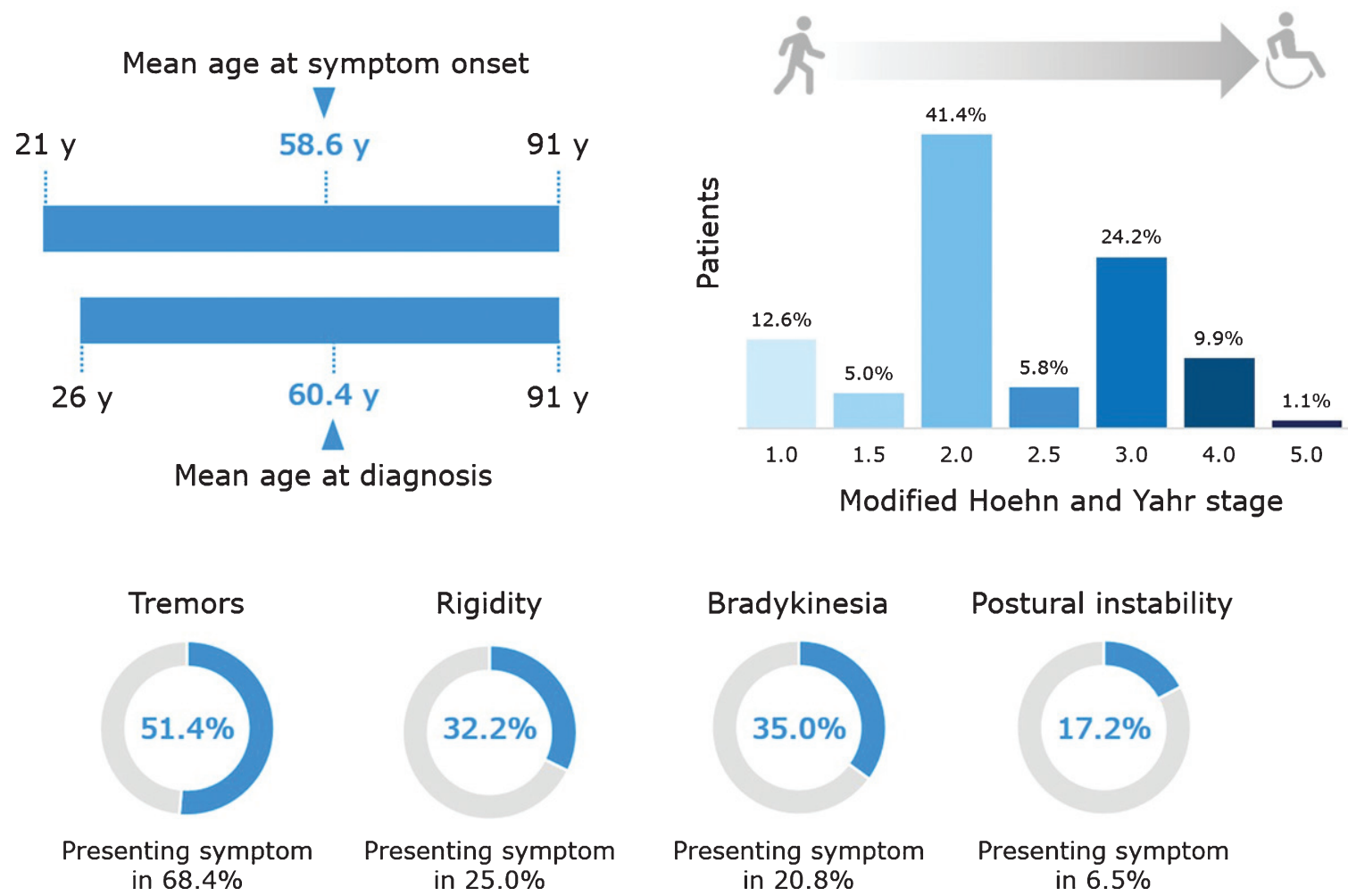

Fig. 3. Key clinical characteristics of patients in the Quebec Parkinson Network cohort. Data are presented for the 1070 patients with PD, except the following for which data were not available for all patients: age at symptom onset $(n=518)$, age at diagnosis $(n=921)$, modified Hoehn and Yahr stage $(n=637)$, postural instability $(n=697)$, tremor as presenting symptom $(n=863)$, rigidity as presenting symptom $(n=748)$, bradykinesia as presenting symptom $(n=787)$, and postural instability as presenting symptom $(n=724)$.

statistically significant differences in the prevalence of presenting symptoms (symptoms reported by the patients at the onset of the diseases) between male and female patients.

\section{Comparison between PD with and without probable RBD}

Since it was previously reported that RBD may represent specific subtypes of PD [15], we compared PD+RBD $(n=381)$ patients to PD-RBD $(n=570)$ patients. Table 3 details the results of the analysis. After Bonferroni correction for multiple comparisons (set the cut-of $p$ value on 0.0028 ) and after adjustment for sex and disease duration, higher frequencies of dyskinesia, fluctuations, postural hypotension and hallucinations were associated with PD+RBD (Table 3). Constipation remained nominally more common in PD+RBD after correction for sex and disease duration but not statistically significant after Bonferroni correction.

\section{Analysis of symptoms by age groups and disease duration}

To examine whether specific symptoms are associated with age groups and disease duration, we performed the following analyses: a) Comparison between EOPD $(n=108)$ and LOPD $(n=410$, Table 4); b) Comparison by current age groups (see Methods for details, Supplementary Table 4); and c) Comparison by disease duration ( $<5$ years, $n=194$; $6-10$ years, $n=163 ;>10$ years, $n=159$, Supplementary Table 5).

Patients with EOPD had a higher rate of dyskinesia compared to LOPD $(53.9 \%$ vs $32.4 \%$, respectively, $p<0.001$, statistically significant after Bonferroni correction for multiple comparisons), as well as fluctuations $(46.9 \%$ vs $30.9 \%, p=0.003$, not statistically significant after Bonferroni correction) and falls ( $23.1 \%$ vs $15.1 \%, p=0.047$, not statistically significant after Bonferroni correction). The frequency of postural instability was higher among LOPD compared to EOPD (24.9\% and $15.0 \%$, respectively, 
Table 2

Sex stratified analysis of PD symptoms in the Quebec Parkinson Network

\begin{tabular}{lccc}
\hline Symptom & \multicolumn{2}{c}{ Patients $(\%)$} & $P$-value \\
\cline { 2 - 3 } & $n=691$ & $\begin{array}{c}\mathrm{a} \\
\end{array}$ & $n=379$ \\
\\
\cline { 2 - 3 } Presenting symptoms & & \\
Tremor & $339 / 691(49.1)$ & $211 / 379(55.7)$ & $0.038^{\mathrm{b}}$ \\
Rigidity & $225 / 691(32.6)$ & $120 / 379(31.7)$ & 0.763 \\
Bradykinesia & $247 / 691(35.7)$ & $128 / 379(33.8)$ & 0.518 \\
Postural instability & $72 / 439(16.4)$ & $48 / 257(18.7)$ & 0.443 \\
Asymmetry & $451 / 691(65.3)$ & $280 / 379(73.9)$ & $0.004^{\mathrm{b}}$ \\
Other symptoms during disease course & & & \\
Dyskinesia & $223 / 515(43.3)$ & $171 / 320(53.4)$ & $0.004^{\mathrm{c}}$ \\
Fluctuations & $206 / 489(42.1)$ & $129 / 296(43.6)$ & 0.690 \\
Freezing of gait & $109 / 691(15.80)$ & $64 / 379(16.9)$ & 0.636 \\
Falls & $80 / 691(11.6)$ & $57 / 379(15.0)$ & 0.105 \\
Postural hypotension & $36 / 506(7.1)$ & $34 / 283(12.0)$ & $0.020^{\mathrm{b}}$ \\
Constipation & $140 / 509(27.5)$ & $89 / 291(30.6)$ & 0.354 \\
Anxiety & $292 / 547(53.4)$ & $200 / 318(62.9)$ & $0.006^{\mathrm{b}}$ \\
Depression & $216 / 531(40.7)$ & $140 / 308(45.5)$ & 0.177 \\
Hallucinations & $110 / 518(21.2)$ & $65 / 296(22.0)$ & 0.809 \\
Impulsive or compulsive behavior & $4 / 488(0.8)$ & $5 / 283(1.8)$ & 0.238 \\
Other psychiatric disorders & $5 / 485(1.0)$ & $1 / 276(0.4)$ & 0.316 \\
Cognitive impairment & $183 / 564(32.4)$ & $102 / 313(32.6)$ & 0.966 \\
Dementia & $15 / 429(3.5)$ & $8 / 255(3.1)$ & 0.801 \\
Sleep disorders & $254 / 488(52.0)$ & $146 / 284(51.4)$ & 0.864 \\
RBD & $269 / 608(44.2)$ & $112 / 343(32.7)$ & $<0.001^{\mathrm{b}}$ \\
\hline
\end{tabular}

RBD, Rapid eye movement (REM) sleep behavior disorder. ${ }^{\text {a Bonferroni correction for }}$ multiple comparisons set the cut-off $p$ value for statistical significance on 0.0025 . ${ }^{\text {b }}$ Not significant after adjustment for disease duration. ${ }^{\mathrm{c}}$ Significant after adjustment for age and disease duration.

Table 3

Analysis of symptoms stratified by RBD status

\begin{tabular}{lccc}
\hline Symptom & \multicolumn{2}{c}{ Patients (\%) } & $P$-value \\
\cline { 2 - 3 } & $\begin{array}{c}\text { Non-RBD } \\
n=570\end{array}$ & $\begin{array}{c}\text { RBD } \\
\end{array}$ & \\
\hline Presenting symptoms & & & \\
$\quad$ Tremor & $323 / 570(56.7)$ & $201 / 381(52.8)$ & 0.235 \\
Rigidity & $187 / 570(32.8)$ & $141 / 381(37.0)$ & 0.182 \\
Bradykinesia & $201 / 570(35.3)$ & $153 / 381(40.2)$ & 0.126 \\
Postural instability & $67 / 426(15.7)$ & $49 / 256(19.1)$ & 0.251 \\
Asymmetry & $425 / 570(74.6)$ & $270 / 381(70.9)$ & 0.208 \\
Other symptoms during disease course & & & \\
Dyskinesia & $193 / 481(40.1)$ & $180 / 321(56.1)$ & $<0.001^{\mathrm{c}}$ \\
Fluctuations & $167 / 461(36.2)$ & $156 / 301(51.8)$ & $<0.001^{\mathrm{c}}$ \\
Freezing of gait & $98 / 570(17.2)$ & $70 / 381(18.4)$ & 0.640 \\
Falls & $67 / 570(11.8)$ & $64 / 381(16.8)$ & $0.027^{\mathrm{b}}$ \\
Postural hypotension & $31 / 479(6.5)$ & $37 / 286(12.9)$ & $0.002^{\mathrm{c}}$ \\
Constipation & $121 / 482(25.1)$ & $102 / 294(34.7)$ & $0.004^{\mathrm{c}}$ \\
Anxiety & $279 / 507(55.0)$ & $191 / 326(58.6)$ & 0.312 \\
Depression & $190 / 491(38.7)$ & $149 / 315(47.3)$ & $0.016^{\mathrm{b}}$ \\
Hallucinations & $82 / 483(17.0)$ & $87 / 302(28.8)$ & $<0.001^{\mathrm{c}}$ \\
Impulsive or compulsive behavior & $3 / 464(0.6)$ & $6 / 285(2.1)$ & 0.075 \\
Other psychiatric disorders & $2 / 460(0.4)$ & $4 / 280(1.4)$ & 0.144 \\
Cognitive impairment & $162 / 463(35.0)$ & $114 / 305(37.4)$ & 0.500 \\
Dementia & $12 / 418(2.9)$ & $9 / 251(3.6)$ & 0.608 \\
\hline
\end{tabular}

RBD, Rapid eye movement (REM) sleep behavior disorder. ${ }^{a}$ Bonferroni correction for multiple comparisons set the cut-off $p$ value for statistical significance on 0.0028 . ${ }^{\text {b }}$ Not significant after adjustment for disease duration. ${ }^{\mathrm{c}}$ Significant after adjustment for sex and disease duration. 
Table 4

Comparison of symptoms between early- and late-onset PD

\begin{tabular}{lccc}
\hline Symptom & \multicolumn{2}{c}{ Patients $(\%)$} & $P$-value \\
\cline { 2 - 3 } & $\begin{array}{c}\text { Late onset } \\
n=410\end{array}$ & $\begin{array}{c}\text { Early onset } \\
n=108\end{array}$ & \\
\hline Presenting symptoms & & & \\
$\quad$ Tremor & $269 / 410(65.6)$ & $65 / 108(60.2)$ & 0.295 \\
Rigidity & $143 / 410(34.9)$ & $46 / 108(42.6)$ & 0.138 \\
Bradykinesia & $146 / 410(35.6)$ & $38 / 108(35.2)$ & 0.935 \\
Postural instability & $90 / 362(24.9)$ & $15 / 100(15.0)$ & $0.037^{\mathrm{c}}$ \\
Asymmetry & $302 / 410(73.7)$ & $80 / 108(74.1)$ & 0.930 \\
Other symptoms during disease course & & & \\
Dyskinesia & $119 / 367(32.4)$ & $55 / 102(53.9)$ & $<0.001^{\mathrm{b}}$ \\
Fluctuations & $111 / 359(30.9)$ & $46 / 98(46.9)$ & $0.003^{\mathrm{b}}$ \\
Freezing of gait & $86 / 410(21.0)$ & $28 / 108(25.9)$ & 0.269 \\
Falls & $62 / 410(15.1)$ & $25 / 108(23.1)$ & $0.047^{\mathrm{b}}$ \\
Postural hypotension & $34 / 395(8.6)$ & $11 / 104(10.6)$ & 0.533 \\
Constipation & $139 / 392(35.5)$ & $32 / 104(30.8)$ & 0.371 \\
Anxiety & $206 / 402(51.2)$ & $57 / 106(53.8)$ & 0.643 \\
Depression & $150 / 401(37.4)$ & $42 / 105(40.0)$ & 0.626 \\
Hallucinations & $61 / 398(15.3)$ & $22 / 105(21.0)$ & 0.167 \\
Impulsive or compulsive behavior & $2 / 387(0.5)$ & $0 / 102(0.0)$ & 0.467 \\
Other psychiatric disorders & $1 / 382(0.3)$ & $1 / 102(1.0)$ & 0.315 \\
Cognitive impairment & $112 / 338(33.1)$ & $25 / 84(29.8)$ & 0.554 \\
Dementia & $5 / 335(1.5)$ & $1 / 93(1.1)$ & 0.762 \\
Sleep disorders & $184 / 392(46.9)$ & $57 / 105(54.3)$ & 0.181 \\
RBD & $141 / 395(35.7)$ & $38 / 105(36.2)$ & 0.925 \\
\hline
\end{tabular}

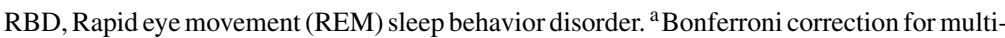
ple comparisons set the cut-off $p$ value for statistical significance on 0.0026 . ${ }^{\mathrm{b}}$ Not significant after adjustment for disease duration. ${ }^{\mathrm{c}}$ Significant after adjustment for age.

$p=0.037$, not statistically significant after Bonferroni correction). As expected, age of patients was associated with higher Hoehn \& Yahr stage $(p<0.001)$, even after adjustment for AAO, disease duration and sex. Furthermore, older patients had significantly higher rates of constipation $(p<0.001$, Supplementary Table 4), and cognitive impairment $(p<0.001)$, which remained statistically significant after adjustment for disease duration and after Bonferroni correction for multiple comparisons. Longer disease duration was associated with higher rates of dyskinesia ( $p<0.001$, Supplementary Table 5), fluctuations ( $p=0.001)$, freezing of gait $(p<0.001)$, falls $(p<0.001)$, hallucinations $(p<0.001)$ and cognitive impairment $(p<0.001)$, all statistically significant after adjustment for age, age at onset and sex, and after correction for multiple comparisons.

\section{DISCUSSION}

\section{The Quebec Parkinson Network cohort as an open-access, representative PD cohort}

Our preliminary analyses demonstrate that the QPN cohort is a typical, representative PD cohort from tertiary and academic centers, comparable to previous reports describing different clinical and epidemiological characteristics and correlations within similar PD cohorts. For example, the QPN cohort's epidemiological characteristics such as sex distribution, age at onset, diagnosis and enrollment are in line with previously published cohorts [16-18]. The associations between different clinical variables (e.g., disease duration and cognitive decline [19, 20], RBD and hallucinations $[21,22]$, etc.) further exemplify that this cohort is typical for academic centers. Of note, since now web-based recruitment to QPN is available, almost one-third of QPN patients are from community-based clinics. However, availability of web access and knowledge may also somewhat bias the recruitment. Another limitation is that data collected by questionnaire and from patients' medical files (when accessible) may be less accurate.

The QPN (http://rpq-qpn.ca/en/) collects data and samples through 4 main channels: the QPN Questionnaire (see Supplementary Material), medical records of patients enrolled in the QPN Participant Registry, research projects facilitated by the QPN (described later), and the NeurO C-BIGR (see Methods). In addition, other data are being collected 
for all patients, or in some cases for subgroups of patients, such as genetic, imaging, neuropsychological and neuropsychiatric data etc. Genetic data are being collected from all patients for whom DNA is available (currently $>800$ patients, out of $>1,200$ patients that have been enrolled by the completion of this paper), including genotyping using a custom single nucleotide polymorphism (SNP) array with $>700,000$ SNPs (Backbone of the OmniExpress array with custom content of the NeuroX SNP-chop, Illumina, Inc.), and targeted next generation sequencing of $\sim 50$ PD-related genes, including GBA, LRRK2, SNCA, MAPT and others [23-25]. For a large number of patients (currently 400), peripheral blood mononuclear cells (PBMCs) are being collected, and iPSCs and neuronal models are being generated by the MNI-iPSC platform (https://mniopenresearch.org/articles/3-1) for specific studies as required. These data and bio-samples are being made available by applying for specific research projects through the QPN website and obtaining approval from the QPN scientific committee and the tissue and data committee of the Neuro C-BIGR. The success of our model may encourage funding of similar initiative, preferably in underrepresented parts of the world where clinical and genetic data on PD patients are less available.

\section{Initiating studies with the Quebec Parkinson Network data and samples}

The QPN has three main research axes: 1) Clinical and Treatment Research, 2) Non-Motor Symptoms, and 3) Molecular and Cellular Biology. Since its creation in June 2013, the QPN has supported more than 60 research projects involving participants from the registry, leading to more than 30 peer-reviewed publications to date. QPN-facilitated research projects are initiated when an investigator requests to use the registry, to access existing data or to enroll QPN patients for their study and collect additional data or samples for their study. The QPN selects patients from the registry for participation in the study based on the inclusion and exclusion criteria provided by the investigator. If the investigator approves inclusion of the participant in their study, they may contact the participant. An initial contact form, indicating whether or not the participant will be included in the study, is submitted by the investigator to the QPN and this information is documented by the QPN for each participant. Participants who are not included in the study remain eligible to be selected for other studies. Participants may be involved in several studies simultaneously; however, the QPN ensures that participants already taking part in a study are not selected for another study deemed incompatible with the first. In this way, the registry provides access to patients for investigators conducting research in PD. As the QPN Participant Registry continues to grow, the QPN is consolidating data collected through the questionnaire, patient medical records, and QPNfacilitated research studies, such as NeurO C-BIGR, in a centralized, open-access database (LORIS [Longitudinal Online Research and Imaging System]) [26-28]. All epidemiological, clinical, neuroimaging, neuropsychological, and biological data will then be made publicly accessible to promote data sharing throughout the PD research community, ultimately leading to new research projects and analyses. Applications for data and for new studies can be made through the QPN website (http://rpq-qpn.ca/en/).

\section{Recent studies and trials facilitated by the Quebec Parkinson Network}

A number of recently published studies using QPN patients in the Clinical and Treatment Research axis have investigated wearable movement detection technology that would allow neurologists to monitor patients beyond the clinic. Data collected from inertial measurement units (IMUs) worn by patients with PD were used to assess motor symptoms including tremor, bradykinesia, and freezing of gait, as well as drug-induced dyskinesia [29]. Using this inertial sensor technology, algorithms were developed that can detect and segment movements in patients performing a Timed Up and Go task [30] or carrying out activities of daily living in a simulated living space [31]. IMU data were also used to identify a turning signature that discriminates between older-age adults and older-age patients with PD, as well as between patients in ON and OFF medication states [32]. The algorithms are currently being tested in a smartphone app that will be used to detect and measure tremor and bradykinesia in a QPN-supported project.

Of importance to advancing treatment for patients with PD and PD-related disorders, the QPN has assisted with participant recruitment for 2 ongoing clinical trials. The MOVES-PD trial is a phase II global study to assess the dynamics, efficacy, and safety of GZ/SAR402671 (Genzyme/Sanofi), a small molecule inhibitor of glucosylceramide synthase [33], in patients with early-stage PD who carry a mutation in the gene encoding glucocerebrosidase 
(GBA) (NCT02906020). A second trial is assessing the efficacy, safety, tolerability, and pharmacokinetics of ABBV-8E12 (AbbVie), a humanized anti-Tau antibody [34], in patients with progressive supranuclear palsy (PSP), an atypical parkinsonian syndrome (NCT02985879).

The second QPN research axis focuses on the non-motor symptoms of PD through the use of neuroimaging and neuropsychological evaluations. Several published studies have identified potential predictors of specific non-motor symptoms, including depressive symptoms, cognitive impairment, and dementia. The severity of depressive symptoms in non-demented patients with PD was shown to be associated with higher dosages of levodopa [35] and with higher rates of cortical thinning [36]. Cognitive deficits in patients with PD were correlated with RBD [37], a longer history of hypertension and higher pulse pressure [38], and a history of smoking [39]. A study of dementia in patients with PD identified age, male sex, baseline RBD, orthostatic hypotension, and mild cognitive impairment (MCI) as significant predictors of dementia, and the co-existence of RBD, MCI, and orthostatic hypotension at baseline was found to be the strongest determinant for the development of dementia [40]. A recent study suggested that transcranial magnetic stimulation may improve cognition in PD patients [41]. Ongoing projects in this research axis are continuing to investigate cognitive function in patients with PD, as well as auditory, speech, and olfactory functions.

The third QPN research axis is Molecular and Cellular Biology. The majority of publications from this axis are studies on the genetics of PD. Targeted next generation sequencing of candidate genes in patients with PD and healthy controls has supported roles for specific genetic variants in the pathogenesis of the disease, such as variants in GCHI [42], $M A P T$ [23], and SMPD1 [43], and demonstrated lack of association with other genes [44-46]. Furthermore, recruiting patients from the QPN Participant Registry provides an opportunity to access a large number of patients who are of French-Canadian ancestry, which was critical for the recent identification of a founder mutation in the $G B A$ gene in French-Canadian patients with PD or RBD [25]. Genome-wide association study (GWAS) data from QPN patients have also been used for recent largescale genetic analyses of mitochondria-related genes and GWAS of AAO in PD $[47,48]$. The GWAS data are currently being used to accurately determine the ancestry of all participants, and these data will be made available for the users of QPN data. Ongoing projects in this axis aim to identify novel genetic determinants of PD and PD-related disorders, and to study genetic models in dopaminergic neurons and midbrain organoids derived from QPN PD patients.

\section{Future directions and the Canadian Open Parkinson's Network}

The QPN's success in building a robust patient registry with associated epidemiological and clinical data and in establishing standardized processes for facilitating research projects has served as the model for the creation of a Canadian national PD network. The recently funded Canadian Open Parkinson Network (C-OPN) will involve 8 major movement disorder centres across 4 Canadian provinces: the Hotchkiss Brain Institute and Movement Disorders program at the University of Calgary; the McGill Parkinson Program at the MNI and Montreal General Hospital (McGill University); L'Unité des Troubles du Mouvement André Barbeau at the Université de Montréal; La Clinique des Troubles du Mouvement du Centre Hospitalier Universitaire de Québec at the CHU de Québec-Université Laval; the Parkinson Research Consortium (University of Ottawa); the Movement Disorders Centre at Toronto Western Hospital (University of Toronto); the Movement Disorders Program at the University of Alberta; and the Pacific Parkinson's Research Centre at the University of British Columbia. C-OPN aims to create an open-access panel of patient-derived PD stem cell lines with associated clinical and imaging data, in partnership with the Open Science iPSC Platform and C-BIGR at the MNI. Similarly to QPN, COPN has 3 major research initiatives. First, C-OPN will establish a national registry of patients with PD and PD-related disorders containing comprehensive longitudinal clinical information to facilitate patient recruitment for large-scale projects or clinical trials. Second, C-OPN will set up an anonymized database for neuropsychological information, imaging data, and second-generation wearable device data. Third, C-OPN will store biological samples in an open biorepository from which genetic and cellular samples and data will be processed, curated, and made available to the scientific community in Canada and beyond. The goals of C-OPN are to find new technological platforms for imaging and wearable data, identify biomarkers and platforms for drug discovery, and ultimately develop novel stratification methods based on multidimensional data to better predict 
disease trajectory and inform treatment strategies for PD.

\section{ACKNOWLEDGMENTS}

We thank the participants in the Quebec Parkinson Network and their families. The Quebec Parkinson Network is funded by a grant from Fonds de recherche du Québec - Santé (FRQS). The Canadian Open Parkinson Network is funded by a grant from Brain Canada and Parkinson Canada. ZGO is supported by the Fonds de recherche du Québec Santé (FRQS) Chercheurs-boursiers award given in collaboration with Parkinson Quebec, and by the Young Investigator Award from Parkinson Canada. FC is a recipient of a Researcher Chair from the Fonds de recherche du Québec santé (FRQS) providing salary support and operating funds. J. D.-O. is receiving salary support from FRQS and Parkinson Québec. JFG holds a Canada Research Chair in Cognitive Decline in Pathological Aging. JBP was partially funded by NIH-NIBIB P41 EB019936 (ReproNim) NIH-NIMH R01 MH083320 (CANDIShare) and NIH 5U24 DA039832 (NIF), as well as the Canada First Research Excellence Fund, awarded to McGill University for the Healthy Brains for Healthy Lives initiative. OM receives funding from the Tourmaline Chair in Parkinson's Canada, the Canada Research Chair in Non-Motor Symptoms in Parkinson's Disease and a Platform grant from Brain Canada-Parkinson's Canada. EAF is supported by a Foundation grant from the Canadian Institutes of Health Research (CIHR; FDN-154301) and a Canada Research Chair Tier 1 (CRC; \#232176).

\section{CONFLICT OF INTEREST}

ZGO reports consultation fees from Lysosomal Therapeutics Inc. (LTI), Idorsia, Denali, Prevail Therapeutics, Inception Sciences (Ventus), all outside the submitted work. AD received research grants from Flamel Ireland Limited, Pfizer, Canopy Growth, Biron as well as honoraria from speaking engagements from UCB and Biogen. None of the financial disclosures is relevant to the submitted work. RBP reports personal fees from Takeda, Roche/Prothena, Teva Neurosciences, Novartis Canada, Biogen, Boehringer Ingelheim, Theranexus, GE HealthCare, Jazz Pharmaceuticals, Abbvie, Jannsen, and Otsuko, all outside the submitted work.
All other authors have no conflict of interests to report.

\section{SUPPLEMENTARY MATERIAL}

The supplementary material is available in the electronic version of this article: http://dx.doi.org/10. 3233/JPD-191755.

\section{REFERENCES}

[1] de Lau LM, Breteler MM (2006) Epidemiology of Parkinson's disease. Lancet Neurol 5, 525-535.

[2] Kowal SL, Dall TM, Chakrabarti R, Storm MV, Jain A (2013) The current and projected economic burden of Parkinson's disease in the United States. Mov Disord 28, 311-318.

[3] Poewe W, Seppi K, Tanner CM, Halliday GM, Brundin P, Volkmann J, Schrag AE, Lang AE (2017) Parkinson disease. Nat Rev Dis Primers 3, 17013.

[4] Cilia R, Tunesi S, Marotta G, Cereda E, Siri C, Tesei S, Zecchinelli AL, Canesi M, Mariani CB, Meucci N, Sacilotto G, Zini M, Barichella M, Magnani C, Duga S, Asselta R, Solda G, Seresini A, Seia M, Pezzoli G, Goldwurm S (2016) Survival and dementia in GBA-associated Parkinson's disease: The mutation matters. Ann Neurol 80, 662-673.

[5] Gan-Or Z, Bar-Shira A, Mirelman A, Gurevich T, Kedmi M, Giladi N, Orr-Urtreger A (2010) LRRK2 and GBA mutations differentially affect the initial presentation of Parkinson disease. Neurogenetics 11, 121-125.

[6] Kim CY, Alcalay RN (2017) Genetic forms of Parkinson's disease. Semin Neurol 37, 135-146.

[7] Saunders-Pullman R, Mirelman A, Alcalay RN, Wang C, Ortega RA, Raymond D, Mejia-Santana H, Orbe-Reilly M, Johannes BA, Thaler A, Ozelius L, Orr-Urtreger A, Marder KS, Giladi N, Bressman SB, LRRK2 Ashkenazi Jewish Consortium (2018) Progression in the LRRK2-Asssociated Parkinson Disease Population. JAMA Neurol 75, 312-319.

[8] Sardi SP, Cedarbaum JM, Brundin P (2018) Targeted therapies for Parkinson's disease: From genetics to the clinic. Mov Disord 33, 684-696.

[9] Fereshtehnejad SM, Zeighami Y, Dagher A, Postuma RB (2017) Clinical criteria for subtyping Parkinson's disease: Biomarkers and longitudinal progression. Brain 140, 19591976.

[10] Lawton M, Ben-Shlomo Y, May MT, Baig F, Barber TR, Klein JC, Swallow DMA, Malek N, Grosset KA, Bajaj N, Barker RA, Williams N, Burn DJ, Foltynie T, Morris HR, Wood NW, Grosset DG, Hu MTM (2018) Developing and validating Parkinson's disease subtypes and their motor and cognitive progression. J Neurol Neurosurg Psychiatry 89, 1279-1287.

[11] Chen-Plotkin AS, Albin R, Alcalay R, Babcock D, Bajaj V, Bowman D, Buko A, Cedarbaum J, Chelsky D, Cookson MR, Dawson TM, Dewey R, Foroud T, Frasier M, German D, Gwinn K, Huang X, Kopil C, Kremer T, Lasch S, Marek K, Marto JA, Merchant K, Mollenhauer B, Naito A, Potashkin J, Reimer A, Rosenthal LS, Saunders-Pullman R, Scherzer CR, Sherer T, Singleton A, Sutherland M, Thiele I, van der Brug M, Van Keuren-Jensen K, Vaillancourt D, Walt D, West A, Zhang J (2018) Finding useful biomarkers for Parkinson's disease. Sci Transl Med 10, eaam6003. 
[12] Poupon V, Seyller A, Rouleau GA (2017) The Tanenbaum Open Science Institute: Leading a paradigm shift at the Montreal Neurological Institute. Neuron 95, 1002-1006.

[13] Postuma RB, Berg D, Stern M, Poewe W, Olanow CW, Oertel W, Obeso J, Marek K, Litvan I, Lang AE, Halliday G, Goetz CG, Gasser T, Dubois B, Chan P, Bloem BR, Adler CH, Deuschl G (2015) MDS clinical diagnostic criteria for Parkinson's disease. Mov Disord 30, 1591-1601.

[14] Postuma RB, Arnulf I, Hogl B, Iranzo A, Miyamoto T, Dauvilliers Y, Oertel W, Ju YE, Puligheddu M, Jennum P, Pelletier A, Wolfson C, Leu-Semenescu S, Frauscher B, Miyamoto M, Cochen De Cock V, Unger MM, Stiasny-Kolster K, Fantini ML, Montplaisir JY (2012) A single-question screen for rapid eye movement sleep behavior disorder: A multicenter validation study. Mov Disord 27, 913-916.

[15] Fereshtehnejad SM, Romenets SR, Anang JB, Latreille V, Gagnon JF, Postuma RB (2015) New clinical subtypes of Parkinson disease and their longitudinal progression: A prospective cohort comparison with other phenotypes. JAMA Neurol 72, 863-873.

[16] Gan-Or Z, Amshalom I, Kilarski LL, Bar-Shira A, GanaWeisz M, Mirelman A, Marder K, Bressman S, Giladi N, Orr-Urtreger A (2015) Differential effects of severe vs mild GBA mutations on Parkinson disease. Neurology 84, 880887.

[17] Lesage S, Anheim M, Condroyer C, Pollak P, Durif F, Dupuits C, Viallet F, Lohmann E, Corvol JC, Honore A, Rivaud S, Vidailhet M, Durr A, Brice A, French Parkinson's Disease Genetics Study Group (2011) Large-scale screening of the Gaucher's disease-related glucocerebrosidase gene in Europeans with Parkinson's disease. Hum Mol Genet 20, 202-210.

[18] Sidransky E, Nalls MA, Aasly JO, Aharon-Peretz J, Annesi G, Barbosa ER, Bar-Shira A, Berg D, Bras J, Brice A, Chen CM, Clark LN, Condroyer C, De Marco EV, Durr A, Eblan MJ, Fahn S, Farrer MJ, Fung HC, Gan-Or Z, Gasser T, Gershoni-Baruch R, Giladi N, Griffith A, Gurevich T, Januario C, Kropp P, Lang AE, Lee-Chen GJ, Lesage S, Marder K, Mata IF, Mirelman A, Mitsui J, Mizuta I, Nicoletti G, Oliveira C, Ottman R, Orr-Urtreger A, Pereira LV, Quattrone A, Rogaeva E, Rolfs A, Rosenbaum H, Rozenberg R, Samii A, Samaddar T, Schulte C, Sharma M, Singleton A, Spitz M, Tan EK, Tayebi N, Toda T, Troiano AR, Tsuji S, Wittstock M, Wolfsberg TG, Wu YR, Zabetian CP, Zhao Y, Ziegler SG (2009) Multicenter analysis of glucocerebrosidase mutations in Parkinson's disease. N Engl J Med 361, 1651-1661.

[19] Aarsland D, Andersen K, Larsen JP, Perry R, WentzelLarsen T, Lolk A, Kragh-Sorensen P (2004) The rate of cognitive decline in Parkinson disease. Arch Neurol 61, 1906-1911.

[20] Aarsland D, Muniz G, Matthews F (2011) Nonlinear decline of mini-mental state examination in Parkinson's disease. Mov Disord 26, 334-337.

[21] Onofrj M, Thomas A, D'Andreamatteo G, Iacono D, Luciano AL, Di Rollo A, Di Mascio R, Ballone E, Di Iorio A (2002) Incidence of RBD and hallucination in patients affected by Parkinson's disease: 8-year follow-up. Neurol Sci 23Suppl 2, S91-94.

[22] Pacchetti C, Manni R, Zangaglia R, Mancini F, Marchioni E, Tassorelli C, Terzaghi M, Ossola M, Martignoni E, Moglia A, Nappi G (2005) Relationship between hallucinations, delusions, and rapid eye movement sleep behavior disorder in Parkinson's disease. Mov Disord 20, 1439-1448.
[23] Li J, Ruskey JA, Arnulf I, Dauvilliers Y, Hu MTM, Hogl B, Leblond CS, Zhou S, Ambalavanan A, Ross JP, Bourassa CV, Spiegelman D, Laurent SB, Stefani A, Charley Monaca C, Cochen De Cock V, Boivin M, Ferini-Strambi L, Plazzi G, Antelmi E, Young P, Heidbreder A, Labbe C, Ferman TJ, Dion PA, Fan D, Desautels A, Gagnon JF, Dupre N, Fon EA, Montplaisir JY, Boeve BF, Postuma RB, Rouleau GA, Ross OA, Gan-Or Z (2018) Full sequencing and haplotype analysis of MAPT in Parkinson's disease and rapid eye movement sleep behavior disorder. Mov Disord 33, 1016-1020.

[24] Ouled Amar Bencheikh B, Ruskey JA, Arnulf I, Dauvilliers Y, Monaca CC, De Cock VC, Gagnon JF, Spiegelman D, Hu MTM, Hogl B, Stefani A, Ferini-Strambi L, Plazzi G, Antelmi E, Young P, Heidbreder A, Mollenhauer B, Sixel-Doring F, Trenkwalder C, Oertel W, Montplaisir JY, Postuma RB, Rouleau GA, Gan-Or Z (2018) LRRK2 protective haplotype and full sequencing study in REM sleep behavior disorder. Parkinsonism Relat Disord 52, 98-101.

[25] Ruskey JA, Zhou S, Santiago R, Franche LA, Alam A, Ronciere L, Spiegelman D, Fon EA, Trempe JF, Kalia LV, Postuma RB, Dupre N, Rivard GE, Assouline S, Amato D, Gan-Or Z (2018) The GBA p.Trp378Gly mutation is a probable French-Canadian founder mutation causing Gaucher disease and synucleinopathies. Clin Genet 94, 339-345.

[26] Das S, Glatard T, MacIntyre LC, Madjar C, Rogers C, Rousseau ME, Rioux P, MacFarlane D, Mohades Z, Gnanasekaran R, Makowski C, Kostopoulos P, Adalat R, Khalili-Mahani N, Niso G, Moreau JT, Evans AC (2016) The MNI data-sharing and processing ecosystem. Neuroimage 124, 1188-1195.

[27] Das S, Glatard T, Rogers C, Saigle J, Paiva S, MacIntyre L, Safi-Harab M, Rousseau ME, Stirling J, Khalili-Mahani N, MacFarlane D, Kostopoulos P, Rioux P, Madjar C, LecoursBoucher X, Vanamala S, Adalat R, Mohaddes Z, Fonov VS, Milot S, Leppert I, Degroot C, Durcan TM, Campbell T, Moreau J, Dagher A, Collins DL, Karamchandani J, BarOr A, Fon EA, Hoge R, Baillet S, Rouleau G, Evans AC (2016) Cyberinfrastructure for open science at the Montreal Neurological Institute. Front Neuroinform 10, 53.

[28] Das S, Zijdenbos AP, Harlap J, Vins D, Evans AC (2011) LORIS: A web-based data management system for multicenter studies. Front Neuroinform 5, 37.

[29] Goubault E, Nguyen HP, Bogard S, Blanchet PJ, Bezard E, Vincent C, Langlois M, Duval C (2018) Cardinal motor features of Parkinson's disease coexist with peak-dose choreic-type drug-induced dyskinesia. J Parkinsons Dis $\mathbf{8}$, 323-331.

[30] Nguyen H, Lebel K, Boissy P, Bogard S, Goubault E, Duval C (2017) Auto detection and segmentation of daily living activities during a Timed Up and Go task in people with Parkinson's disease using multiple inertial sensors. $\mathrm{J} \mathrm{Neu}$ roeng Rehabil 14, 26.

[31] Nguyen H, Lebel K, Bogard S, Goubault E, Boissy P, Duval C (2018) Using inertial sensors to automatically detect and segment activities of daily living in people with Parkinson's disease. IEEE Trans Neural Syst Rehabil Eng 26, 197-204.

[32] Lebel K, Duval C, Nguyen HP, Plamondon R, Boissy P (2018) Cranio-caudal kinematic turn signature assessed with inertial systems as a marker of mobility deficits in Parkinson's disease. Front Neurol 9, 22.

[33] Ashe KM, Budman E, Bangari DS, Siegel CS, Nietupski JB, Wang B, Desnick RJ, Scheule RK, Leonard JP, Cheng SH, Marshall J (2015) Efficacy of enzyme and substrate reduction therapy with a novel antagonist of glucosylceramide synthase for Fabry disease. Mol Med 21, 389-399. 
[34] West T, Hu Y, Verghese PB, Bateman RJ, Braunstein JB, Fogelman I, Budur K, Florian H, Mendonca N, Holtzman DM (2017) Preclinical and clinical development of ABBV-8E12, a humanized anti-tau antibody, for treatment of Alzheimer's disease and other tauopathies. J Prev Alzheimers Dis 4, 236-241.

[35] Hanganu A, Degroot C, Monchi O, Bedetti C, MejiaConstain B, Lafontaine AL, Chouinard S, Bruneau MA (2014) Influence of depressive symptoms on dopaminergic treatment of Parkinson's disease. Front Neurol 5, 188.

[36] Hanganu A, Bruneau MA, Degroot C, Bedetti C, MejiaConstain B, Lafontaine AL, Chouinard S, Monchi O (2017) Depressive symptoms in Parkinson's disease correlate with cortical atrophy over time. Brain Cogn 111, 127-133.

[37] Jozwiak N, Postuma RB, Montplaisir J, Latreille V, Panisset M, Chouinard S, Bourgouin PA, Gagnon JF (2017) REM sleep behavior disorder and cognitive impairment in Parkinson's disease. Sleep 40, doi: 10.1093/sleep/zsx101

[38] Doiron M, Langlois M, Dupre N, Simard M (2018) The influence of vascular risk factors on cognitive function in early Parkinson's disease. Int J Geriatr Psychiatry 33, 288297.

[39] Doiron M, Dupre N, Langlois M, Provencher P, Simard M (2017) Smoking history is associated to cognitive impairment in Parkinson's disease. Aging Ment Health 21, 322-326.

[40] Anang JB, Nomura T, Romenets SR, Nakashima K, Gagnon JF, Postuma RB (2017) Dementia predictors in Parkinson disease: A validation study. J Parkinsons Dis 7, 159-162.

[41] Trung J, Hanganu A, Jobert S, Degroot C, Mejia-Constain B, Kibreab M, Bruneau MA, Lafontaine AL, Strafella A, Monchi O (2019) Transcranial magnetic stimulation improves cognition over time in Parkinson's disease. Parkinsonism Relat Disord 66, 3-8.

[42] Rudakou U, Ouled Amar Bencheikh B, Ruskey JA, Krohn L, Laurent SB, Spiegelman D, Liong C, Fahn S, Waters C, Monchi O, Fon EA, Dauvilliers Y, Alcalay RN, Dupre N, Gan-Or Z (2019) Common and rare GCH1 variants are associated with Parkinson's disease. Neurobiol Aging 73, 231 e231-231 e236.

[43] Alcalay RN, Mallett V, Vanderperre B, Tavassoly O, Dauvilliers Y, Wu RYJ, Ruskey JA, Leblond CS, Ambalavanan A, Laurent SB, Spiegelman D, Dionne-Laporte A, Liong C, Levy OA, Fahn S, Waters C, Kuo SH, Chung WK, Ford B, Marder KS, Kang UJ, Hassin-Baer S, Greenbaum L, Trempe JF, Wolf P, Oliva P, Zhang XK, Clark LN, Langlois M, Dion PA, Fon EA, Dupre N, Rouleau GA, Gan-Or Z (2019) SMPD1 mutations, activity, and alpha-synuclein accumulation in Parkinson's disease. Mov Disord 34, 526535 .
[44] Blauwendraat C, Kia DA, Pihlstrom L, Gan-Or Z, Lesage S, Gibbs JR, Ding J, Alcalay RN, Hassin-Baer S, Pittman AM, Brooks J, Edsall C, Chung SJ, Goldwurm S, Toft M, Schulte C, International Parkinson's Disease Genomics Consortium (IPDGC), COURAGE-PD Consortium, Hernandez D, Singleton AB, Nalls MA, Brice A, Scholz SW, Wood NW (2018) Insufficient evidence for pathogenicity of SNCA His50Gln (H50Q) in Parkinson's disease. Neurobiol Aging 64, 159 e $155-159$ e 158.

[45] Blauwendraat C, Reed X, Kia DA, Gan-Or Z, Lesage S, Pihlstrom L, Guerreiro R, Gibbs JR, Sabir M, Ahmed S, Ding J, Alcalay RN, Hassin-Baer S, Pittman AM, Brooks J, Edsall C, Hernandez DG, Chung SJ, Goldwurm S, Toft M, Schulte C, Bras J, Wood NW, Brice A, Morris HR, Scholz SW, Nalls MA, Singleton AB, Cookson MR, COURAGEPD (Comprehensive Unbiased Risk Factor Assessment for Genetics and Environment in Parkinson's Disease) Consortium, the French Parkinson's Disease Consortium, and the International Parkinson's Disease Genomics Consortium (IPDGC) (2018) Frequency of loss of function variants in LRRK2 in Parkinson disease. JAMA Neurol 75, 1416-1422.

[46] Ross JP, Dupre N, Dauvilliers Y, Strong S, Dionne-Laporte A, Dion PA, Rouleau GA, Gan-Or Z (2017) RIC3 variants are not associated with Parkinson's disease in FrenchCanadians and French. Neurobiol Aging 53, 194 e199-194 e111.

[47] Billingsley KJ, Barbosa IA, Bandres-Ciga S, Quinn JP, Bubb VJ, Deshpande C, Botia JA, Reynolds RH, Zhang D, Simpson MA, Blauwendraat C, Gan-Or Z, Gibbs JR, Nalls MA, Singleton A, International Parkinson's Disease Genomics C, Ryten M, Koks S (2019) Mitochondria function associated genes contribute to Parkinson's Disease risk and later age at onset. NPJ Parkinsons Dis $\mathbf{5}, 8$.

[48] Blauwendraat C, Heilbron K, Vallerga CL, Bandres-Ciga S, von Coelln R, Pihlstrom L, Simon-Sanchez J, Schulte C, Sharma M, Krohn L, Siitonen A, Iwaki H, Leonard H, Noyce AJ, Tan M, Gibbs JR, Hernandez DG, Scholz SW, Jankovic J, Shulman LM, Lesage S, Corvol JC, Brice A, van Hilten JJ, Marinus J, andMe Research T, Eerola-Rautio J, Tienari P, Majamaa K, Toft M, Grosset DG, Gasser T, Heutink P, Shulman JM, Wood N, Hardy J, Morris HR, Hinds DA, Gratten J, Visscher PM, Gan-Or Z, Nalls MA, Singleton AB, International Parkinson's Disease Genomics Consortium (IPDGC) (2019) Parkinson's disease age at onset genome-wide association study: Defining heritability, genetic loci, and alpha-synuclein mechanisms. Mov Disord 34, 866-875. 